MRS Advances (C) 2016 Materials Research Society

DOI: $10.1557 /$ adv.2016.484

\title{
Effects of Synthesis Parameters on CVD Molybdenum Disulfide Growth
}

Gustavo A. Lara Saenz ${ }^{1}$, Chandan Biswas ${ }^{1,2}$, Hisato Yamaguchi ${ }^{3}$,Claudia Narvaez Villarrubia ${ }^{3}$, Aditya D. Mohite ${ }^{3}$ and Anupama B. Kaul ${ }^{* 1,2}$

${ }^{1}$ Electrical and Computer Engineering Department, University of Texas, El Paso, TX, U.S.A.

${ }^{2}$ Metallurgical, Materials and Biomedical Engineering Department, University of Texas, El Paso, TX, 79968, U.S.A.

${ }^{3}$ Materials Physics and Applications Division, Los Alamos National Laboratory, Los Alamos, NM, 87545, U.S.A.

*E-mail: akaul@utep.edu

\begin{abstract}
Since the isolation of graphene, a monolayer of $\mathrm{sp}^{2}$-bonded carbon atoms arranged in a hexagonal lattice, two-dimensional (2D) layered materials have attracted a great deal of attention due to their outstanding mechanical, optical and electronic properties. The research areas of interest for these new materials include exploring their novel properties, developing scalable approaches to synthesize these materials, and integrating them into a new generation of nanodevices. The utilization of 2D materials in devices has many advantages, which includes scaled materials to the limit of atomic-scale membranes, and the potential to form device structures on flexible and transparent substrates, among others. Transition metal dichalcogenides (TMDs) monolayers in particular, have received increasing attention in recent years, especially molybdenum disulfide $\left(\mathrm{MoS}_{2}\right)$, which is one of the most well explored semiconducting materials in the 2D materials system. In this work we present the synthesis of $\mathrm{MoS}_{2}$ using chemical vapor deposition (CVD), where we have varied the synthesis parameters and compared the structure and quality of the CVD synthesized $\mathrm{MoS}_{2}$. At the same time, we have compared the characteristics with those obtained for mechanically exfoliated flakes from the bulk $\mathrm{MoS}_{2}$ crystal. The $\mathrm{MoS}_{2}$ quality has been analyzed using Raman spectroscopy.
\end{abstract}

\section{INTRODUCTION}

One of the most investigated transition metal dichalcogenides (TMDs) is $\mathrm{MoS}_{2}$ which possess some interesting properties such as the transition from indirect band gap to direct band gap from bulk to single layer with a thickness of $\sim 6.5 \AA$ [1], photosensitivity for detector and photovoltaic applications [2], as well as its promise in flexible electronics. The production of $2 \mathrm{H}$ polytype $\mathrm{MoS}_{2}$ via chemical vapor deposition (CVD) is an area of research interest that allows the scalable synthesis of large area, high quality, and single layer of this material. There are four different CVD synthesis approaches used to manufacture monolayer $\mathrm{MoS}_{2}$ : vapor phase reaction, sulfurization of metal oxide, transport and recrystallization of powder, and thermolysis of precursor compound containing Mo and S atoms; the last approach has been the most applied and studied in the 
literature. In previous reports, the parameters used for the synthesis have not been captured in great detail and the growth process is not yet fully understood.

In this work it is reported the synthesis parameters used for two CVD mechanisms: the sulfurization of metal oxide and the reaction of precursors in the vapor phase. In the sulfurization route, first the molybdenum trioxide is evaporated then reduced with sulfur vapor to molybdenum dioxide. After the reduction, $\mathrm{MoO}_{2}$ rhomboidal crystals are grown on the substrate. Next, the sulfurization occurs and the $\mathrm{MoS}_{2}$ grows on top of the oxide crystals (Equation 1) [3,4].

$$
\mathrm{MoO}_{2}+3 \mathrm{~S} \rightarrow \mathrm{MoS}_{2}+\mathrm{SO}_{2}
$$

In the second method, the reaction of both precursors occur in vapor phase. First the reduction of the $\mathrm{MoO}_{3}$ precursor (Equation 2), then the reaction to form $\mathrm{MoS}_{2}$ to finally grow on the substrate (Equation 3) [5].

shows the phase diagram of molybdenum - oxygen - sulfur and the paths for the reaction for $\mathrm{MoS}_{2}$.

$$
\begin{gathered}
2 \mathrm{MoO}_{3}+x \mathrm{~S} \rightarrow 2 \mathrm{MoO}_{3-x}+x \mathrm{SO}_{2} \\
2 \mathrm{MoO}_{3-x}+(7-x) \mathrm{S} \rightarrow 2 \mathrm{MoS}_{2}+(3-x) \mathrm{SO}_{2}
\end{gathered}
$$

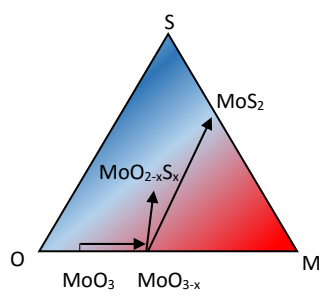

Figure 1. Phase diagram of molybdenum, oxygen, and sulfur, showing the paths of the reduction of $\mathrm{MoO}_{3}$ to the sulfurization to create molybdenum oxisulfides and the final reaction to form $\mathrm{MoS}_{2}$.

\section{EXPERIMENTAL}

The synthesis parameters that affect the growth during the CVD synthesis are: the pressure, mass load of precursors, flow rate of transport gas, temperature of precursors and substrate, position of precursors, substrate, time of process, and substrate treatment. In the subsequent section, we describe the role of each of these factors. 
The most common substrate used for the CVD synthesis of $\mathrm{MoS}_{2}$ is the $\mathrm{Si} / \mathrm{SiO}_{2}$ with a thickness of $270 \mathrm{~nm}$, which is the optimal thickness for obtaining the highest optical contrast to identify MoS2 monolayers under the optical microscope reaching a positive contrast of around $25-30 \%$ [6]. This substrate is frequently utilized for $\mathrm{MoS}_{2}$ CVD growth despite the disadvantages that it entails. The $\mathrm{MoS}_{2}$ crystallites grow in random orientations, since the nucleation points are sparsely located over the substrate. In addition, the area of single domains is small and the crystals overlap each other, and the triangular crystals grow at the vicinity of the edges, on dust particles, and scratches (see Figure 2 for examples of these cases). The synthesis on $\mathrm{SiO}_{2}$ can be improved with the application aromatic molecules used as seed promoters such as PTAS (perylene-3,4,9,10tetracarboxylic acid tetrapotassium salt), r-GO (reduced graphene oxide), and PTCDA (3,4,9,10perylene-tetracarboxylicacid-dianhydride), in which the PTAS is reported with the best results by Ling et al [7]. In addition, the substrate treatment is crucial for the quality of the growth. The largest domain size has been reported a size $\sim 120 \mu \mathrm{m}$ through the treatment of the $\mathrm{SiO}_{2} / \mathrm{Si}$ substrate with bath ultrasonication, followed by two hours of piranha solution, and finishing the cleaning process with an $\mathrm{O}_{2}$ plasma. This process increases the domain sites without the application of any seed promoter [8]. Other substrates such as mica, quartz, and sapphire have been demonstrated to obtain better growth results thanks to the lattice matching with $\mathrm{MoS}_{2}$, high thermal stability, flat surface, and chemical inertness that assists the precursor migration to improve the thickness uniformity [9].

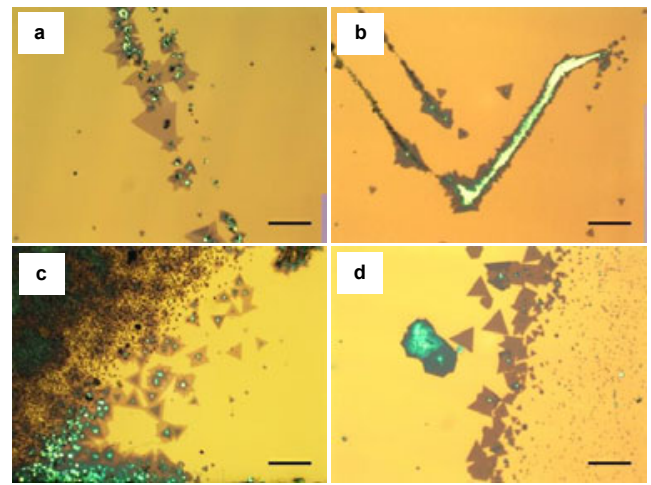

Figure 2. $\mathrm{MoS}_{2}$ triangular crystals on $\mathrm{Si} / \mathrm{SiO}_{2}$ substrate grown in a) dust particles, b) scratches, and c) vicinity of edge. d) Random crystal growth orientation of doimains. The bright spots corresponds to the original nucleation sites of the crystal. The scale bar is $60 \mu \mathrm{m}$ for the four optical images.

The temperature of the precursors is one of the most important parameters of the synthesis, this is controlled by the position of the boats and the temperature profile is set in the furnace. The temperatures required for sulfurization $\left(\sim 850-950{ }^{\circ} \mathrm{C}\right)$ are higher than the temperature for reduction of $\mathrm{MoO}_{3}\left(\sim 650-850^{\circ} \mathrm{C}\right)$ [10]. Additionally, low temperatures in precursors results in a low vapor concentration in the reaction chamber. Another item that is affected by the process temperature is the substrate, which influences the adsorption and desorption of the $\mathrm{MoO}_{3}$ and $\mathrm{MoS}_{2}$ 
clusters and hence the nucleation density and domain size of the $\mathrm{MoS}_{2}$ flakes. On the other hand, the pressure during the growth process alters the crystallinity, the density, and the single domain size of the $\mathrm{MoS}_{2}$. The pressure in the reaction chamber is increased by evaporation of the sulfur. As the pressure increases, the vaporization of Mo is reduced and consequently the vapor concentration that leads to the change of shape of the single domain from hexagons to triangles, to three point stars [5]. Furthermore, the flow rate of the transport gas is an important parameter to control the deposition rate. Nevertheless, high flow rate results in unstable growth and at the same time creates defects such as dendritic morphologies due to the crystal growth driven more by reaction kinetics than equilibrium thermodynamic processes [11]. The mass load of the precursors is directly related to the their vapor concentration which at the same time is related to the morphology of the domains with different terminations, Mo-zigzag or S-zigzag [11]. Low load reduces the deposition rate that can be compensated by increasing the flow rate of the transport gas. When the sulfur supply is insufficient, it grows rectangular oxisulfides instead of $\mathrm{MoS}_{2}$.

In our experiments, the synthesis of $\mathrm{MoS}_{2}$ was done in $\mathrm{SiO}_{2} / \mathrm{Si}(270 \mathrm{~nm}$ of thickness oxide) substrates. It was treated in ultrasonic bath using acetone, methanol and DI water for 5 minutes each, dehydrated and cleaned with piranha solution for 30 minutes. The precursor utilized are the $\mathrm{MoO}_{3}$ (sigma Aldrich 99.9995\%) and S (sigma Aldrich 99.5\%) with a mass of $0.4 \mathrm{~g}$ and $0.8 \mathrm{~g}$ deposited on alumina boat of dimensions $100 \times 40 \times 18 \mathrm{~mm}$ and $50 \times 20 \times 20 \mathrm{~mm}$ respectively. The $\mathrm{AlO}_{3}$ boats are placed in the CVD system designed by our research group, the $\mathrm{MoO}_{3}$ in the middle, and $\mathrm{S}$ at 36 and $17 \mathrm{~cm}$ from the center (Figure 3) for the two different mechanism of $\mathrm{MoS}_{2}$ synthesis. Subsequently, the reaction chamber (which consist in a quartz tube of $72 \mathrm{~mm}$ of inside diameter and a length of $1.4 \mathrm{~m}$ ) was first vacuumed down to $90 \mathrm{mTorr}$, afterwards it was purged with UHP $\mathrm{N}_{2}$, the transport gas, at $200 \mathrm{sccm}$ for 15 minutes followed by the increasing of pressure up to 760 Torr by closing the outlet valve. Next, the outlet valve was controlled to obtain a stable pressure of 760 Torr inside the tube. Finally, the furnace program was run and the pressure controlled manually by operating the outlet needle valve. At the end of the program, the furnace was naturally cool down to room temperature. After each experiment the boats were cleaned in piranha solution for 30 minutes.

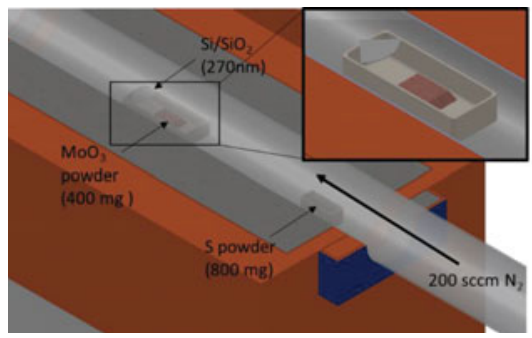

Figure 3. Position of substrate and precursors in alumina boats inside the CVD furnace. Long distance between the precursors assure homogeneous concentration of sulfur vapor. By changing this distance it is modified the route of $\mathrm{MoS}_{2}$ synthesis. 


\section{DISCUSSION}

In the first method, during the sulfurization of $\mathrm{MoO}_{2}$, the furnace was heated up on a rate of 15 ${ }^{\circ} \mathrm{C} / \mathrm{min}$ and the temperature was kept at $850{ }^{\circ} \mathrm{C}$ for 15 minutes. These conditions yielded to the growth of rhomboidal crystals, Sample A. In contrast, in the second mechanism, during the vapor phase reaction, the temperature ramp rate was $17.6^{\circ} \mathrm{C} / \mathrm{min}$ up to 550 , where the sulfur began to evaporate, then the ramp was decreased to $5^{\circ} \mathrm{C} / \mathrm{min}$ until a temperature of $850{ }^{\circ} \mathrm{C}$ was reached for a duration of 15 minutes; this yielded to the synthesis of triangular crystals, Sample B. By changing the position of the sulfur boat $\mathrm{MoS}_{2}$ was synthesized by two different routes, sulfurization $(36 \mathrm{~cm}$ from the center) and vapor phase reaction (17 $\mathrm{cm}$ from the center). The long distance of sulfur boat from the center leads to homogeneous concentration.

The analysis of the two samples obtained by the two different approaches was done using Raman spectroscopy. We have confirmed that monolayer $\mathrm{MoS}_{2}$ was synthesized as in Sample B where the strong vibration modes $\mathrm{E}_{2 \mathrm{~g}}$ and $\mathrm{A}_{1 \mathrm{~g}}$ corresponds to the characteristic Raman peaks of 388.4 and $488.4 \mathrm{~cm}^{-1}$ respectively. This results in a $\Delta \mathrm{k}=20 \mathrm{~cm}^{-1}$ (Figure 4), which agrees well with previous reports for monolayer $\mathrm{MoS}_{2}$ [12]. The Raman spectrum for Sample A shows the peaks that correspond to $\mathrm{MoO}_{2}$ and $\mathrm{MoS}_{2}$ for some rhomboidal flakes and just $\mathrm{MoO}_{2}$ for others, agreeing with previous synthesis reports which involve the sulfurization of $\mathrm{MoO}_{2}[2,3]$.

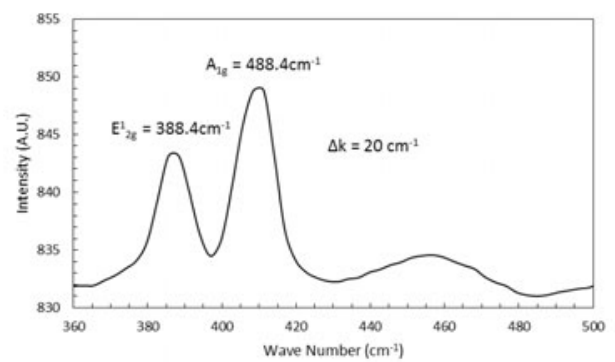

Figure 4. Raman spectrum of Sample B, $\mathrm{MoS}_{2}$ monolayer is confirmed by $\Delta \mathrm{k}=20 \mathrm{~cm}^{-1}$ of the two strong peaks $\mathrm{E}^{1}{ }_{2 \mathrm{~g}}$ and $\mathrm{A}_{1 \mathrm{~g}}$, at 388.4 and $488.4 \mathrm{~cm}^{-1}$ respectively.

\section{CONCLUSIONS}

$\mathrm{MoS}_{2}$ was successfully synthesized by two different routes, sulfurization and vapor phase reaction, on $\mathrm{SiO}_{2} / \mathrm{Si}$ substrates by changing the position of the $\mathrm{S}$ precursor in the reaction chamber. In the second method, we have verified monolayer $2 \mathrm{D} \mathrm{MoS}_{2}$ was formed as confirmed using Raman spectroscopy. Details of our systematic experiments to study how each of the parameters (pressure, flow rate, transport gas, temperature, time, position of precursors and treatment) affects the $\mathrm{MoS}_{2}$ growth by AFM, Raman spectroscopy, TEM, and STEM measurements are presented in reference [13]. In our future work, we will transfer the synthesized $\mathrm{MoO}_{2}$ through the sulfurization process, using poly (methyl methacrylate) (PMMA) to another substrate for a more detailed analysis, which should present an opportunity to integrate these materials into novel electronic and optoelectronic devices. 


\section{ACKNOWLEDGMENT}

Support is acknowledged from the University of Texas System Faculty Science and Technology Acquisition and Retention (STARS) award (EC284802) for the acquisition of equipment in the establishment of the Nanomaterials and Devices Lab (NDL) at the University of Texas, El Paso (UTEP). We also thank the Air Force Office of Scientific Research (grant number FA9550-15-10200) that enabled us to pursue this work.

\section{REFERENCES}

1. B. Radisavljevic, A. Radenovic, J. Brivio, V. Giacometti, and A. Kis, Nat. Nanotechnol., 6, 147-150 (2011).

2. G. A. Lara Saenz, D. Fadil, G. Karapetrov, and A. Kaul, to be submitted, (2016).

3. Y.-C. Lin, W. Zhang, J.-K. Huang, K.-K. Liu, Y.-H. Lee, C.-T. Liang, C.-W. Chu, and L.-J. Li, Nanoscale, 4, 6637 (2012).

4. Y. Zhan, Z. Liu, S. Najmaei, P. M. Ajayan, and J. Lou, Small, 8, 966-971 (2012).

5. S. Najmaei, Z. Liu, W. Zhou, X. Zou, G. Shi, S. Lei, B. I. Yakobson, J.-C. Idrobo, P. M. Ajayan, and J. Lou, Nat. Mater., 12, 754-9 (2013).

6. M. M. Benameur, B. Radisavljevic, J. S. Héron, S. Sahoo, H. Berger, and A. Kis, Nanotechnology, 22, 125706 (2011).

7. X. Ling, Y.-H. Lee, Y. Lin, W. Fang, L. Yu, M. S. Dresselhaus, and J. Kong, Nano Lett., 12, 464-72, (2014).

8. A. M. van der Zande, P. Y. Huang, D. a Chenet, T. C. Berkelbach, Y. You, G.-H. Lee, T. F. Heinz, D. R. Reichman, D. a Muller, and J. C. Hone, Nat. Mater., 12, 554-61 (2013).

9. Y. Yu, C. Li, Y. Liu, L. Su, Y. Zhang, and L. Cao, Sci. Rep., 3, 1866, (2013).

10. Y. H. Lee, X. Q. Zhang, W. Zhang, M. T. Chang, C. Te Lin, K. Di Chang, Y. C. Yu, J. T. W. Wang, C. S. Chang, L. J. Li, and T. W. Lin, Adv. Mater., 24, 2320-2325 (2012).

11. S. Wang, Y. Rong, Y. Fan, M. Pacios, H. Bhaskaran, K. He, and J. H. Warner, Chem. Mater., 26, 6371-6379 (2014).

12. C. Lee, H. Yan, L. E. Brus, T. F. Heinz, J. Hone, and S. Ryu, ACS Nano, 4, 2695-700, (2010).

13. G. A. Lara Saenz, C. Biswas, H. Yamaguchi, C. N. Villarrubia A. Mohite, and A. B. Kaul, manuscript in preparation, to be submitted (2016). 